\title{
Profile Based Side-View Face Authentication using Pose Variants: A Survey Surinder Pal Singh ${ }^{1}$, Ashish Oberoi ${ }^{2}$ \\ ${ }^{1,2}$ (MMEC,MMU-Mullana,India), \\ (1's.p.singh@mmumullana.org, ${ }^{2}$ ashish.oberoi@mmumullana.org)
}

\begin{abstract}
As a widely used biometrics, side face recognition has many advantages such as being a legal act of entering, formed by nature and submitting without resistance or objection. On the other hand, in existing scenarios with constrained environment, pose variation up to side-view positions makes face recognition a challenging work. This paper recognize the research toward pose-invariant face recognition in recent years and to find issues in profile based side-face recognition across poses which are still remain open, such as lack of understanding about subspaces of pose variant images. It compares available databases suitable for different task such as surveillance, criminal identification and house safety applications also.
\end{abstract}

Keywords- Controlled Environment, Face Detection, Feature Extraction, Poses, Occlusions.

\section{INTRODUCTION}

Various biometric technologies, which recognize human have been proposed for user authentication such as fingerprints, hand geometry, retina, iris, and facial images, which are collected from physical measurements of the human body. Face recognition plays a vital role in biometrics for identifying individuals. Face recognition has become the need for the society and investment in security applications are continuously increasing day by day. The problem of face recognition in images has received a considerable amount of attention in recent years due to numerous applications in practice, including person identification, surveillance, and image/video annotation. A variety of face recognition methods has been reported in the literature. Despite the large number of methods for face recognition, their performance remains unsatisfactory for images containing side-view faces. While in applications such as access control via face identification, it is reasonable to look for a front-view face only, applications such as surveillance, criminal identification from a different database, and image/video annotation would greatly benefit by being able to detect side-view faces.[1]

The configuration of generic side-view face recognition is:

(a) Face Detection: The steps of face detection are as follows:

(i) The main purpose of face detection is to determine the information such as whether there exists face and the position, the rotation and the pose of the face in the image. The method of face detection varies, according to the fixed layout of the color or the density of face organ as well as face is determined. So the method based on skin color models and template matching is the important research direction of face detection.

(ii) The face detection method based on template matching chooses full face feature as the matched template, with which the burden of computing of face search is relatively large. However, most human faces are symmetry obviously. So half of the full face-template that is choosing the left half face or the right half face as the template of face matching which can reduce the burden of computing of face search[1].

(b) Feature Extraction: The steps of feature extraction are as follows:

(i) Firstly align the landmarks of the images in the training set to find the average landmarks. Then computing of the transformation between each image. Finally to get the average landmarks and transform images. The fixed sized images a bounding rectangle around the face and crop the image is used for finding the parameters of the rebounding from a rectangle surface and computing the distance between the nasion and the nose tip using the average landmarks and height, width is used for finding distance is multiplied with a constant. Moreover, locate the rectangle using the average nose tip landmark, and crop all images accordingly.

(ii) Now apply some pre-processing to the images to eliminate the effect of background. Landmarks and interpolate are used to estimate the profile. Using this profile information, background is removed and 
gets a cropped image that contains both the shape information and the texture information. Now warp the image by stretching the profile until the edge of the bounding box. After warping, smoothing of the image using a low-pass filter is done . Finally, A circle with its centre in the middle of the right edge of the bounding rectangle and remove the area outside that circle[7].

(c) Face Recognition Methods: The methods are according to the technique used for coping the pose differences between the gallery images and the test images:

(i) Feature-Based Methods: when the input image and the gallery image are in different poses, either the transformation in feature space is learned and applied to extracted features to handle pose variations, or features that are robust to pose variations are used. In identifying people using side-view face images, the categorization of methods are according to their relevancy to our problem like the images are acquired from video, 2D color camera or the data is gathered in unrestricted condition.

(ii) Image-Based Methods: In image-based face recognition techniques, when the input image and the gallery image are in different poses having typical and attractive faces, a new image from either gallery image or input image is synthesized by twisting or with the aid of a 3-Dimension face reconstruction system. So, the pose variation is handled by synthesizing images that contain the same pose as the original image[1][7].

Many of the face recognition approaches are based on geometric patterns, figures and similar location of facial attributes, such as the nose, lips, eyes eyebrows and chin. Face recognition has many advantages such as being tending to intrude without invitation, accordance with the natural behavior of the individual and without giving active response or resistance to the individual. It is very difficult to work on face recognition, since faces are characterized by a variety of shapes, similar (attractive and typical )poses, textures, sizes and textures. There are some of the following problems:

- Pose - A position of the camera can be horizontally, vertically or with different angles so that a face can vary when the image is captured.

- Presence of structural components - There may be an additional feature on the face such as different shapes of spectacles, moustache or beard with different type of shape, color of the hair and textures.

- Facial expression - A face can be smiley, crying, sad or of different moods when the person's face image is captured.

- Occlusion - A face may have the blockage or partially obstructed by someone else or when the image is captured among crowds.

- Image condition - The condition of an image like blur, noising, long distance exposure depends on the lighting and camera characteristics.

Side-view face recognition is having a wide scope of application because when the frontal view portraits are not available then the side-view face can be used as a compatible matching feature for authentication/verification to improve the accuracy. Side-view face recognition is a demanding problem due to the many different and connected parts of human face. There are many implementation area for side-view face recognition techniques such as detecting thief in home, car burglar and also militant. The unfortunate incident and injuries happening in the home as well as external environment are mostly caused by ignoring the risks, busy schedule of the parents, or extraneous threats. Therefore, side-view face recognition can be used to identify and process the critical elements of information that may cause further unfortunate incident or to detect an emergency in time[10].

\section{Profile BaSed Side -VieW FaCe ReCognition ReVIEW}

The problem addressed in this paper is to recognize a side-view face image. There exist two categories of related work: appearance-based and profile-based methods. Appearance-based methods use the side-view face image as input directly. Profile-based methods use the profile extracted from the side-view image.

In the appearance-based methods, there are two different scenarios for side face recognition. In the first one, the probe is the side face and the gallery is the frontal face. This is an extreme situation of face recognition across pose. Detailed literature reviews about face recognition across pose are provided in [11]. However, most methods are only able to work on face images with limited variations in pose and the performance drastically decreases when applied to data with -90 o to 90 o pose change. Blanz et al. [19] proposed face identification across 
different poses based on a 3D morphable model. However, the pre alignment had to be done manually and thus cannot achieve automatic recognition. In the second scenario, both the probe and gallery are side-view face images. This could be considered as an ex- ample of multi-view face recognition. Pentland et al. [20] proposed to extend the popular Eigen face approach on a database of 21 people with poses from $-90^{\circ}$ to $90^{\circ}$. Zhou and Bhanu [22] proposed to apply PCA and multiple discriminate analysis in side-view face recognition. In order to align the side-view face images in both training and test sets, the fiducial points extracted by a curvature-based method were used. Generally, including the texture information of the human face results in more discriminating results. However, in an outdoor situation, the texture of the face is sensitive to illumination variations. The performance of appearance based methods will deteriorate under changing lighting conditions.

In contrast, profile-based methods use the shape information of the human face and are insensitive to the illumination changing. The method proposed in this paper falls under the profile-based recognition category. In the 70's, Harmon et al. [11] published their seminal work in face profile recognition. They extract the geometry features of the face profile to form a 10-dimensional feature vector, according to the positions of 9 fiducially points on the profile. In their later work [10], the number of fiducially points was increased to 11 to form a 17-dimensional feature vector for each profile. Bhanu and Zhou [3] proposed a method using dynamic time warping (DTW) to match face profiles. According to the curvature value of each point on the profile, the pronasle and the nasion points are found and used to crop the face part and discard of the non-face parts. The similarity score between the probe and each profile in the gallery are computed by the DTW based on curvature. Zhou and Bhanu [22] extended this work to face profile recognition in video by constructing a high-resolution side face image from a series of aligned low-resolution side face images. Computing the fiducially point positions based on curvature in these methods is very crucial since it depends on scale-space preprocessing. This is biased towards the size of the profile and the variance of the Gaussian applied to estimate the required scale. Applying the scale-space approach on different profiles does not guarantee the same curvature patterns in all cases. Accurate localization of the fiducially points greatly affects the recognition results. To the best of our knowledge, there is no fiducially point's detection algorithm which is able to work correctly and robustly on large databases. In order to overcome this, registration-based methods have been proposed. Pan et al. [15] presented an experimental comparison for the face profile recognition problem. The authors compared different profile alignment methods, such as tangent based normalization, 2D iterative closest point alignment (ICP) and simulated annealing (SA) alignment.

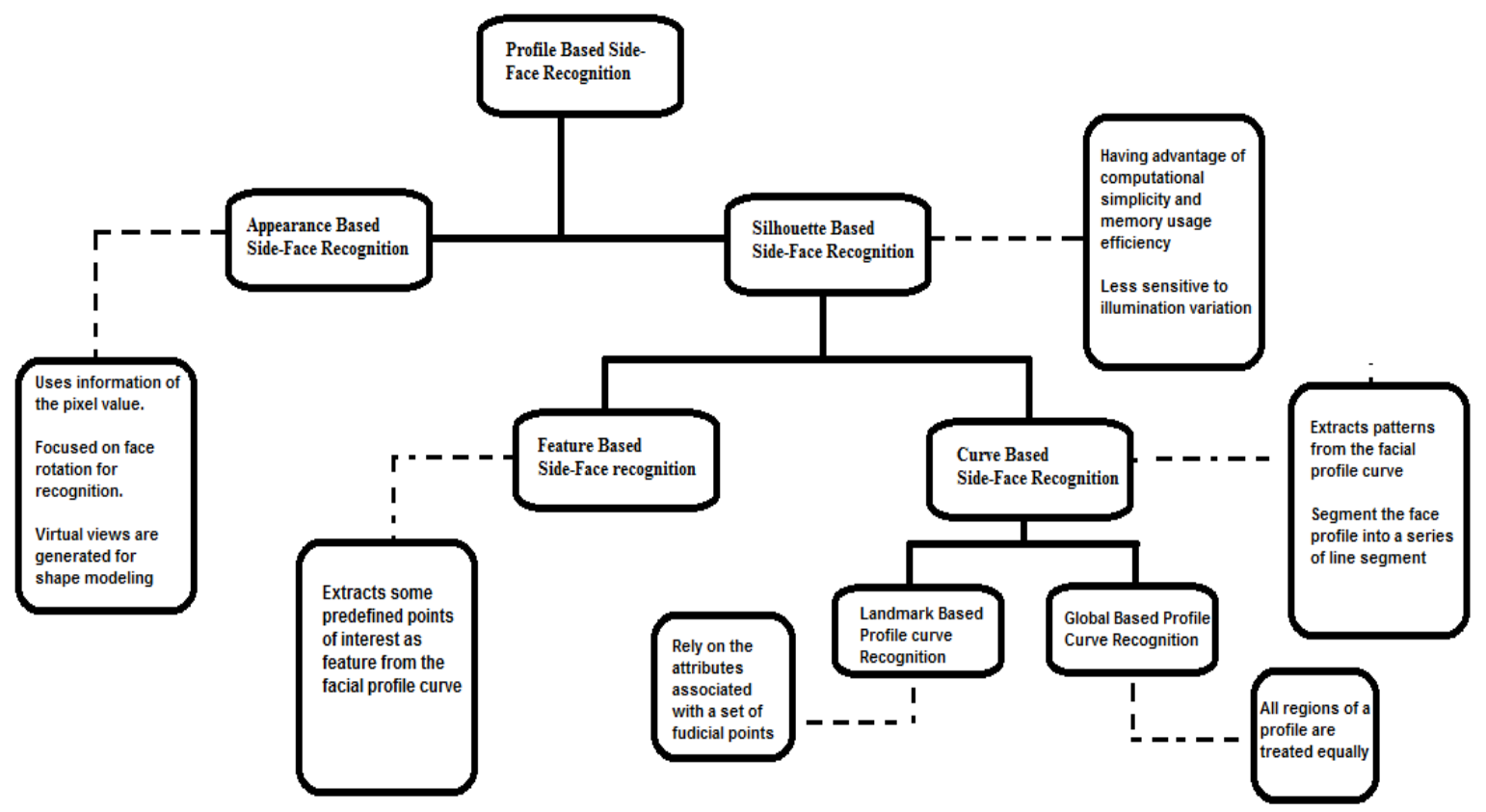

Fig. 1: Classification of Profile Based Side-Face Recognition 
In addition to the side-view face image databases, the profiles extracted from 3D database are also used to evaluate different methods. They concluded that the SA and 2D ICP method achieved the best recognition performance. Gao and Leung [12] proposed a string matching method for face profile recognition. The face profile is first transformed into a series of line segments. Then each line segment is represented by its attributes such as the length, orientation and midpoint. After performing the merge domain string matching method, the distance score of the probe and the gallery profiles were computed. The approach assumes that the two compared profiles have the same curve details and fails whenever occlusion occurs. Wu et al. [13] proposed a face recognition method based on three different curves extracted from the 3D face model. Besides the face profile, they use two horizontal curves of a $3 \mathrm{D}$ face. Recognizing 2D profiles is very important since obtaining the planar profiles from a digital image is much easier and widely applicable than extracting the three curves used in the above technique. In addition to human authentication, facial profile analysis has been applied in many areas (i.e., facial expression analysis [16], and 3D face reconstruction [19]).

\section{DATABASE USED IN LITERATURE REVIEW}

There are a different databases that having side face images with variant poses which also include sideview face images. Many of them are collected in a supervised and controlled environment with steady background, artificial illumination changes, or restricted pose variations. The largest available database for side-view face recognition is CMU-Multi PIE database [9] with 337 subjects and 15 poses. It is an supplement of CMU-PIE database [17], which contains only 68 subjects and 13 poses. Additional database that is used in side-view face recognition research is FERET database having 200 subjects and 9 poses. There are also some databases that are collected in more uncontrolled settings. MMI database [15] is a web-based movements convey the emotional state of an individual expression database including 1500 samples of 19 people. In addition to the video, in some databases 3D captures, or sound files are also provided. UHDB1 database contains sixteen captures of 141 subjects, where the subject is sitting in a car with different poses, and a camera is capturing the scene at a right angle $\left(90^{\circ}\right)$ to the subject. The database also includes five 3D captures of each subject in variant poses. XM2VTS database [20] contains four recordings of 295 subjects taken over a different period for four months.

TABLE I

DATABASE USED IN THE LITERATURE REVIEW

\begin{tabular}{|l|l|l|l|l|}
\hline \multicolumn{1}{|c|}{ Name } & \multicolumn{1}{|c|}{$\begin{array}{c}\text { No. of } \\
\text { Subjects }\end{array}$} & \multicolumn{1}{|c|}{$\begin{array}{c}\text { No of } \\
\text { Images }\end{array}$} & Poses & Highlights \\
\hline CMU-PIE [17] & 68 & 13 & 15 & gray scale, background, lighting, expression, eye positions \\
\hline CMU-MultiPIE [9] & 337 & 15 & 13 & lighting, expression, background, pose \\
\hline FERET [8] & 200 & 9 & 9 & $\begin{array}{l}\text { color images, changes in appearance through time, controlled pose } \\
\text { variation, facial expression }\end{array}$ \\
\hline Bern [6] & 30 & 5 & $\sqrt{ }$ & lighting, expression, background, 3D, sequences \\
\hline XM2VTSDB [14] & 295 & 5 & 4 & very large database, pose, illumination, expression \\
\hline M2VTS [12] & 37 & 19 & 9 & large pose changes, speaking subjects, eye glasses, time change \\
\hline MMI [15] & 19 & 5 & $\sqrt{ }$ & eye glasses, beards, college age, pose \\
\hline UHDB1 [11] & 141 & 17 & 7 & highly varied illumination, eye glasses \\
\hline Bosphorus [16] & 105 & 13 & $\sqrt{ }$ & speaking subjects, eye glasses, time change \\
\hline
\end{tabular}

Each recording contains a speaking head shot and a rotating head shot. The database includes high class or standard color images, video sequences and a 3Dimensional Models. It is an extension of the M2VTS database, which contains voice and motion sequences of 37 people, who have been asked to count from ' 0 ' to ' 9 ' in their 
native language, and rotate their head from left to right. The Bosphorus Database [21] is a recent 3D face database that includes a rich set of expressions like crying, laughing, smiling etc , different angles of poses and various types of occlusions.

\section{Challenges In Side-Face ReCOGNition}

A key goal of computer vision researchers is to create automated profile based side-face recognition systems that can equally or better than human performance. It is imperative that computational researchers know the key findings of side-face recognition by humans.

- Human can recognize side faces in extremely low-resolution images.

- Human ability to tolerate degradations increases with familiarity .

- In the context of human vision, line-drawings appear to be sufficient for recognition purposes.

- Both internal and external facial cuts are important for recognition purposes.

- The nature of cues used: Pigmentation and shape.

- Color cues play a significant role especially when shape and pigment cues are degraded.

- Motion of faces appears to facilitate subsequent recognition

\section{PSYCHOLOGY/NEUROSCIENCE ISSUES IN PROFILE BASED SIDE-FACE RECOGNITION}

Many studies and finding in psychology and neuroscience have a direct relevance to the engineers interested in designing algorithm for machine recognition of side face. Paper summarize findings that are potentially relevant to the design of side face recognition system.

- Effect of lighting changes.

- Moving sequence of the face.

- Facial expression.

- Different view-point and invariant description.

- Distinctiveness(typical faces and attractive faces).

- Ranking of significance of facial feature.

\section{CONCLUSION}

This paper reviews the current side-view face recognition approaches and to recognize people from sideview angles especially in real-world applications such as surveillance systems, smart homes, or any application dealing with identifying people in videos. In such applications, the environment is uncontrolled, and head pose is unrestricted, illumination is varying, and expression may be apparent. This paper compares available databases that are collected in challenging environments and containing real-world scenarios.

\section{REFERENCES}

[1] X. Zhang, and Y. Gao, "Face recognition across pose: A review," Pattern Recognition, 42, $2009,2876-2896$.

[2] Y. Gao, and M. Leung, "Line segment Hausdorff distance on face matching," Pattern Recognition, 35, 2002, 361-371.

[3] B. Bhanu, and X. Zhou, "Face Recognition from Face Profile Using Dynamic Time Warping," Int. Conf. on Pattern Recognition (ICPR), 4, 2004, 499-502.

[4] F. Tsalakanidou, “Use of depth and colour eigen faces for face recognition,” Pattern Recognition Letters, 24, $2003,1427-1435$.

[5] R. Gross, J. Yang, and A. Waibel, "Face Recognition in a Meeting Room," IEEE Int. Conf. on Automatic Face and Gesture Recognition, 294, 2000.

[6] V. Blanz, and T. Vetter, "Face recognition based on fitting a 3D morphable model," IEEE Transactions on PAMI,25, 2003, 10631074.

[7] I.A. Kakadiaris, H. Abdelmunim, W. Yang, and T. Theoharis, "Profile-based face recognition," IEEE Int. Conf. on Automatic Face and Gesture Recognition (FG '08), 2008, 1-8.

[8] K. A. Ghaffary, F. A. Tab and H. Danyali, "Profile-based Face Recognition using the Outline Curve of the Profile Silhouette," IJCA Special Issue on "Artificial Intelligence Techniques - Novel Approaches \& Practical Applications AIT,4, 2011, 38-43.

[9] X. Zhang, Y. Gao and M. Leung, "Recognizing rotated faces from frontal and side views: an approach toward effective use of mugshot databases," IEEE Trans. on Information Forensics and Security,3, 2008,684-697.

[10] G. Pan, L. Zheng, and Z. Wu, "Robust metric and alignment for profile-based face recognition: an experimental comparison," in Proc. Seventh IEEE Workshops on Application of Computer Vision, 1, 2005,117-122.

[11] Y. Gao and M. K.H. Leung, “Human face profile recognition using attributed string,” Pattern Recognition, 35, $2002,353-360$.

[12] Z. Liposcak and S. Loncaric, “A scale-space approach to face recognition from profiles,” in Proc. 8th International Conference on Computer Analysis of Images and Patterns, London, UK, 243-250, 1999. 
IOSR Journal of Computer Engineering (IOSR-JCE)

e-ISSN: 2278-0661, p-ISSN: 2278-8727

[13] H. Wu, P. Zhou and Z. Gao, "An algorithm for automatic side face portrait recognition based on Fourier descriptor," in Proc Information Science and Engineering, ISISE '08,2, 2008,767-772.

[14] G. Wei, D. Li and I. K. Sethi, "Detection of side-view faces in color images”, in Proc. Fifth IEEE Workshop on Applications of Computer Vision, 2000, 79-84.

[15] Agung Harsoyo, Muhamad Chairul Rezi, Pranoto H Rusmin ," Design of Face Recognition System Using Local Binary Pattern and Clahe on Smart Meeting Room System", IEEE 3rd International Conference on System Engineering and Technology, 2013978-983,.

[16] Sihao Ding, Qiang Zhai, Yuan F. Zheng, and Dong Xuan," Side view face Authentication based on wavelet and random forest with subsets",Intelligence and Security Informatics (ISI), 2013,76 - 81.

[17] Pinar Santemiz ,Luuk J. Spreeuwers and Raymond N.J. Veldhuis," Automatic Landmark Detection and Face Recognition for SideView Face Images", Biometrics Special Interest Group (BIOSIG), 2013, 1-4.

[18] Jaeik Jo; Yu Jin Jung; Jaihie Kim,"3D face reconstruction from one side-view face images, " International Conference on Electronics, Information and Communications (ICEIC), 2014,1-2.

[19] Xu Xiao-Ma; Pei Ming-Tao," Multiview Face Retrieval in Surveillance Video by Active Training Sample Collection", Tenth International Conference on Computational Intelligence and Security (CIS),2014, 242 - 246.

[20] Yong Xu, Xuelong Li, Jian Yang, David Zhang, "Integrate the original face image and its mirror image for face recognition", Journal of Neurocomputing ,131, 2014,191-199.

[21] Shervin Rahimzadeh Arashloo, Josef Kittler," Fast pose invariant face recognition using super coupled multi resolution Markov Random Fields on a GPU", Journal of Pattern Recognition, 48(1),2015, 73-85.

[22] Jaeik Jo, Heeseung Choi, Ig-Jae Kim, Jaihie Kim," Single-view-based 3D facial reconstruction method robust against pose variations", Journal of Pattern Recognition, 48(1) , 2015 ,73-85. 